\title{
Twistors, Generalizations and Exceptional Structures
}

\author{
Roldão da Rocha*† \\ IFGW, Unicamp, Campinas (SP), Brazil \\ E-mail: roldao@ifi.unicamp.br
}

Jayme Vaz, Jr.

IMECC, Unicamp, Campinas (SP), Brazil

E-mail: vaz@ime.unicamp.br

This paper is intended to describe twistors via the paravector model of Clifford algebras and to relate such description to conformal maps in the Clifford algebra over $\mathbb{R}^{4,1}$, besides pointing out some applications of the pure spinor formalism. We construct twistors in Minkowski spacetime as algebraic spinors associated with the Dirac-Clifford algebra $\mathbb{C} \otimes C \ell_{1,3}$ using one lower spacetime dimension than standard Clifford algebra formulations, since for this purpose the Clifford algebra over $\mathbb{R}^{4,1}$ is also used to describe conformal maps, instead of $\mathbb{R}^{2,4}$. It is possible to identify, via the pure spinor formalism, the twistor fiber in four, six and eight dimensions, respectively, with the coset spaces $\mathrm{SO}(4) /\left(\mathrm{SU}(2) \times \mathrm{U}(1) / \mathbb{Z}_{2}\right) \simeq \mathbb{C P}^{1}, \mathrm{SO}(6) /\left(\mathrm{SU}(3) \times \mathrm{U}(1) / \mathbb{Z}_{2}\right) \simeq \mathbb{C P}^{3}$ and $\mathrm{SO}(8) /\left(\operatorname{Spin}(6) \times \operatorname{Spin}(2) / \mathbb{Z}_{2}\right)$. The last homogeneous space is closely related to the $\mathrm{SO}(8)$ spinor decomposition preserving $\mathrm{SO}(8)$ symmetry in type IIB superstring theory. Indeed, aside the IIB superstring theory, there is no $\mathrm{SO}(8)$ spinor decomposition preserving $\mathrm{SO}(8)$ symmetry and, in this case, one can introduce distinct coordinates and conjugate momenta only if the $\operatorname{Spin}(8)$ symmetry is broken by a $\operatorname{Spin}(6) \times \operatorname{Spin}(2)$ subgroup of $\operatorname{Spin}(8)$. Also, it is shown how to generalize the Penrose flagpole, illustrating the use of the pure spinor formalism to construct a flagpole that is more general than the Penrose one, which arises when a defined parameter goes to zero. We investigate the relation between this flagpole and the $\mathrm{SO}(2 n) / \mathrm{U}(n)$ twistorial structure, which emerges when one considers the action of a suitable classical group on the set $\Xi$ of all totally isotropic subspaces of $\mathbb{C}^{2 n}$, and an isomorphism from the set of pure spinors to $\Xi$. Finally we point out some relation between twistors fibrations and the classification of compact homogeneous quaternionic-Kähler manifolds (the so-called Wolf spaces), and exceptional Lie structures.

Fourth International Winter Conference on Mathematical Methods in Physics

09 - 13 August 2004

Centro Brasileiro de Pesquisas Fisicas (CBPF/MCT), Rio de Janeiro, Brazil

\footnotetext{
${ }^{*}$ Speaker.

${ }^{\dagger}$ The author thanks CAPES for the financial support.
} 


\section{Introduction}

Nowadays the search for any unified theory that describes the four fundamental interactions demands a deep mathematical background and an interface between physics and mathematics. The relation between superstring theory in twistor spaces [1,2] and the pure spinor formalism [3, 4] has been increasingly and widely investigated $[5,6]$. With the motivation concerning the $\mathrm{SO}(8)$ spinor decomposition that preserves $\mathrm{SO}(8)$ symmetry in type IIB superstring theory [7], among others, it can be shown via the pure spinor formalism the well-known result asserting that a twistor in eight dimensions is an element of the homogeneous space $\mathrm{SO}(8) /\left(\operatorname{Spin}(6) \times \operatorname{Spin}(2) / \mathbb{Z}_{2}\right) \simeq \mathrm{SO}(8) / \mathrm{U}(4)$, and, in $n$ dimensions, an element of $\mathrm{SO}(2 n) / \mathrm{U}(n)$.

The main aim of this paper, besides pointing out some relation between twistors and pure spinors, is to describe conformal maps in Minkowski spacetime as the twisted adjoint representation of $\$ \operatorname{pin}_{+}(2,4)$ (to be precisely defined in Sec. 2) on paravectors ${ }^{1}[8,9]$ of $C \ell_{4,1}$, and to characterize twistors as algebraic spinors ${ }^{2}[4]$ in $\mathbb{R}^{4,1}$. Although some papers have already described twistors using the algebra $\mathbb{C} \otimes C \ell_{1,3} \simeq C \ell_{4,1}[10,11,12]$, the present formulation sheds some new light on the use of the paravector model. This paper is presented as follows: in Sec. 2 we describe conformal transformations using the twisted adjoint representation of the group $\operatorname{SU}(2,2)$ $\simeq \$$ pin $_{+}(2,4)$ on paravectors of $C \ell_{4,1}$. In Sec. 3 twistors, the incidence relation between twistors and the Robinson congruence, via multivectors and the paravector model of $\mathbb{C} \otimes C \ell_{1,3} \simeq C \ell_{4,1}$, are introduced. We show explicitly how our results can be led to the well-established ones of Keller [12], and consequently to the classical formulation introduced by Penrose [13, 14]. It is also described how one can obtain twistors as elements of $\mathrm{SO}(2 n) / \mathrm{U}(n)$ via pure spinors. Finally in Sec. 4 we link twistor theory to Lie exceptional structures.

\section{Conformal compactification and the paravector model}

Given a vector space, endowed with a metric $g$ of signature $p-q$, and denoted by $\mathbb{R}^{p, q}$, consider the injective map [9] $\mathbb{R}^{p, q} \ni x \mapsto(x, g(x, x), 1)=(x, \lambda, \mu) \in \mathbb{R}^{p+1, q+1}$. The image of $\mathbb{R}^{p, q}$ under this map is a subset of the Klein absolute $x \cdot x-\lambda \mu=0$. This map induces an injective map from the conformal compactification $\left(S^{p} \times S^{q}\right) / \mathbb{Z}_{2}$ of $\mathbb{R}^{p, q}$ to the projective space $\mathbb{R}^{p+1, q+1}$.

The conformal group $\operatorname{Conf}(p, q)$ is isomorphic to the quotient group $\mathrm{O}(p+1, q+1) / \mathbb{Z}_{2}$ [9], and since the group $\mathrm{O}(p+1, q+1)$ has four components, then $\operatorname{Conf}(p, q)$ has two (if $p$ or $q$ are even) or four components (otherwise) $[9,15]$. Taking the case when $p=1$ and $q=3$, the group $\operatorname{Conf}(1,3)$ has four components, and the component $\operatorname{Conf}_{+}(1,3)$ connected to the identity is the Möbius group ${ }^{3}$ of $\mathbb{R}^{1,3}$. Besides, the orthochronous connected component is denoted by $\operatorname{SConf}_{+}(1,3)$. Consider a basis $\left\{\varepsilon_{\breve{A}}\right\}_{\breve{A}=0}^{5}$ of $\mathbb{R}^{2,4}$ and a basis $\left\{E_{A}\right\}_{A=0}^{4}$ of $\mathbb{R}^{4,1}$. This last basis can be obtained from $\left\{\varepsilon_{\breve{A}}\right\}$ if the isomorphism $E_{A} \mapsto \varepsilon_{A} \varepsilon_{5}$ is defined.

Given $\phi$ an element of the Clifford algebra $C \ell_{p, q}$ over $\mathbb{R}^{p, q}$, the reversion of $\phi$ is defined and denoted by $\tilde{\phi}=(-1)^{[k / 2]} \phi$ ( $[k]$ expresses the integer part of $k$ ), while the graded involution acting

\footnotetext{
${ }^{1} \mathrm{~A}$ paravector of the Clifford algebra $C \ell_{p, q}$ is an element of $\mathbb{R} \oplus \mathbb{R}^{p, q}$.

${ }^{2}$ Algebraic spinors are elements of a minimal lateral ideal of a Clifford algebra.

${ }^{3}$ All Möbius maps are composition of rotations, translations, dilations and inversions [16].
} 
on $\phi$ is defined by $\hat{\phi}=(-1)^{k} \phi$. The Clifford conjugation $\bar{\phi}$ of $\phi$ is given by the reversion composed with the main automorphism.

If we take a vector $\alpha=\alpha^{\breve{A}} \varepsilon_{\breve{A}} \in \mathbb{R}^{2,4}$, a paravector $\mathfrak{b} \in \mathbb{R} \oplus \mathbb{R}^{4,1} \hookrightarrow C \ell_{4,1}$ can be obtained as $\mathfrak{b}=\alpha \varepsilon_{5}=\alpha^{A} E_{A}+\alpha^{5}$. From the periodicity theorem ${ }^{4}$ [17] we have the isomorphism $C \ell_{4,1} \simeq$ $C \ell_{1,1} \otimes C \ell_{3,0} \simeq M(2, \mathbb{C}) \otimes C \ell_{3,0}$, where $M(2, \mathbb{C})$ denotes the group of $2 \times 2$ matrices with complex entries. For $\mathrm{i}=1,2,3$ the isomorphism from $C \ell_{4,1}$ to $C \ell_{3,0}$ is given explicitly by $E_{i} \mapsto E_{i} E_{0} E_{4}:=\mathbf{e}_{i}$, where $\left\{\mathbf{e}_{i}\right\}$ denotes a basis of $\mathbb{R}^{3}$. Defining $E_{ \pm}:=\frac{1}{2}\left(E_{4} \pm E_{0}\right)$, we can write $\mathfrak{b}=\alpha^{5}+\left(\alpha^{0}+\alpha^{4}\right) E_{+}+$ $\left(\alpha^{4}-\alpha^{0}\right) E_{-}+\alpha^{i} \mathbf{e}_{i} E_{4} E_{0}$, and then it is possible, if we represent $E_{+}=\left(\begin{array}{ll}0 & 0 \\ 1 & 0\end{array}\right)$ and $E_{-}=\left(\begin{array}{ll}0 & 1 \\ 0 & 0\end{array}\right)$, to write $\mathfrak{b}=\left(\begin{array}{cc}\alpha^{5}+\alpha^{i} \mathbf{e}_{i} & \alpha^{4}-\alpha^{0} \\ \alpha^{0}+\alpha^{4} & \alpha^{5}-\alpha^{i} \mathbf{e}_{i}\end{array}\right)$. The vector $\alpha \in \mathbb{R}^{2,4}$ is in the Klein absolute, and so $\alpha^{2}=0$. Besides, we assert that $\mathfrak{b}$ is in the Klein absolute if and only if $\alpha$ is. Indeed, denoting $\lambda=\alpha^{4}-\alpha^{0}$ and $\mu=\alpha^{4}+\alpha^{0}$, if $\mathfrak{b} \overline{\mathfrak{b}}=0$, the matrix element $(\mathfrak{b} \overline{\mathfrak{b}})_{11}$ is given by

$$
(\mathfrak{b} \overline{\mathfrak{b}})_{11}=x \bar{x}-\lambda \mu=0,
$$

where $x:=\left(\alpha^{5}+\alpha^{i} \mathbf{e}_{i}\right) \in \mathbb{R} \oplus \mathbb{R}^{3} \hookrightarrow C \ell_{3,0}$. Choosing $\mu=1$ then $\lambda=x \bar{x}$, and this choice is responsible for a projective description. Also, the paravector $\mathfrak{b} \in \mathbb{R} \oplus \mathbb{R}^{4,1}$ can be rewritten as $\mathfrak{b}=\left(\begin{array}{cc}x & x \bar{x} \\ 1 & \bar{x}\end{array}\right)$. From eq.(2.1) we obtain $\left(\alpha^{5}+\alpha^{i} \mathbf{e}_{i}\right)\left(\alpha^{5}-\alpha^{i} \mathbf{e}_{i}\right)=\left(\alpha^{4}-\alpha^{0}\right)\left(\alpha^{4}+\alpha^{0}\right)$ from where $\left(\alpha^{5}\right)^{2}+\left(\alpha^{0}\right)^{2}-\left(\alpha^{1}\right)^{2}-\left(\alpha^{2}\right)^{2}-\left(\alpha^{3}\right)^{2}-\left(\alpha^{4}\right)^{2}=0$, showing that $\alpha$ is indeed in the Klein absolute.

Now consider an element $g \in \mathrm{SU}(2,2) \simeq \$ \operatorname{pin}_{+}(2,4):=\left\{g \in C \ell_{4,1} \mid g \bar{g}=1\right\}$. From the periodicity theorem, it can be represented as $g=\left(\begin{array}{ll}a & c \\ b & d\end{array}\right)$, where $a, b, c, d \in C \ell_{3,0}$.

In order to perform a rotation of the paravector $\mathfrak{b}$, we can use the twisted adjoint representation $\hat{\sigma}: \$ \operatorname{pin}_{+}(2,4) \rightarrow \mathrm{SO}_{+}(2,4)$, defined by its action on paravectors by $\hat{\sigma}(g)(\mathfrak{b})=g \mathfrak{b} \hat{g}^{-1}=g \mathfrak{b} \tilde{g}$. In terms of matrix representations (with entries in $\left.C \ell_{3,0}\right)$, the group $\$ \operatorname{pin}_{+}(2,4)$ acts on paravectors $\mathfrak{b}$ as $g \mathfrak{b} \tilde{g}=\left(\begin{array}{ll}a & c \\ b & d\end{array}\right)\left(\begin{array}{ll}x & \lambda \\ \mu & \bar{x}\end{array}\right)\left(\begin{array}{ll}\bar{d} & \bar{c} \\ \bar{b} & \bar{a}\end{array}\right)$. Fixing $\mu=1, \mathfrak{b}$ is mapped on $\left(\begin{array}{ll}a & c \\ b & d\end{array}\right)\left(\begin{array}{cc}x & x \bar{x} \\ 1 & \bar{x}\end{array}\right)\left(\begin{array}{ll}\bar{d} & \bar{c} \\ \bar{b} & \bar{a}\end{array}\right)=\Delta\left(\begin{array}{cc}x^{\prime} & x^{\prime} \bar{x}^{\prime} \\ 1 & \bar{x}^{\prime}\end{array}\right)$, where $x^{\prime}=(a x+c)(b x+d)^{-1} \in \mathbb{R} \oplus \mathbb{R}^{3}$ and $\Delta=(b x+d)(\overline{b x+d}) \in \mathbb{R}$. In this sense the spacetime conformal maps are rotations in $\mathbb{R} \oplus \mathbb{R}^{4,1}$, performed by the twisted adjoint representation, just given above. All the spacetime conformal maps are expressed respectively by the following matrices $[9,16,18]$ :

\begin{tabular}{||r||r||r||}
\hline \hline Conformal Map & Explicit Map & Matrix of \$pin $(2,4)$ \\
\hline \hline Translation & $x \mapsto x+h, h \in \mathbb{R} \oplus \mathbb{R}^{3}$ & $\left(\begin{array}{ll}1 & \\
0 & 1\end{array}\right)$ \\
\hline Dilation & $x \mapsto \rho x, \rho \in \mathbb{R}$ & $\left(\begin{array}{cc}\sqrt{\rho} & 0 \\
0 & 1 / \sqrt{\rho}\end{array}\right)$ \\
\hline Rotation & $x \mapsto \mathfrak{g} x \hat{\mathfrak{g}}^{-1}, \mathfrak{g} \in \$ \operatorname{pin}_{+}(1,3)$ & $\left(\begin{array}{cc}\mathfrak{g} & 0 \\
0 & \mathfrak{g}\end{array}\right)$ \\
\hline Inversion & $x \mapsto-\bar{x}$ & $\left(\begin{array}{cc}0 & -1 \\
1 & 0\end{array}\right)$ \\
\hline Transvection & $x \mapsto x+x(h x+1)^{-1}, h \in \mathbb{R} \oplus \mathbb{R}^{3}$ & $\left(\begin{array}{ll}1 & 0 \\
h & 1\end{array}\right)$ \\
\hline \hline
\end{tabular}

\footnotetext{
${ }^{4}$ The periodicity theorem of Clifford algebras asserts that $C \ell_{p+1, q+1} \simeq C \ell_{1,1} \otimes C \ell_{p, q}$.
} 
This index-free algebraic formulation allows to trivially generalize the conformal maps of $\mathbb{R}^{1,3}$ to the ones of $\mathbb{R}^{p, q}$, if the periodicity theorem of Clifford algebras is used. The homomorphisms $\$ \operatorname{pin}_{+}(2,4) \simeq \mathrm{SU}(2,2) \stackrel{2-1}{\longrightarrow} \mathrm{SO}_{+}(2,4) \stackrel{2-1}{\longrightarrow} \mathrm{SConf}_{+}(1,3)$ are explicitly constructed in [19].

The generators of $\operatorname{Conf}(1,3)$ are expressed, using a basis $\left\{\gamma_{\mu}\right\} \in C \ell_{1,3}$ and denoting the volume element of $\mathbb{R}^{1,3}$ by $\gamma_{5}=\gamma_{0} \gamma_{1} \gamma_{2} \gamma_{3}$, as $P_{\mu}=\frac{1}{2}\left(\gamma_{\mu}+i \gamma_{\mu} \gamma_{5}\right), K_{\mu}=-\frac{1}{2}\left(\gamma_{\mu}-i \gamma_{\mu} \gamma_{5}\right), D=\frac{1}{2} i \gamma_{5}$, and $M_{\mu v}=\frac{1}{2}\left(\gamma_{\nu} \wedge \gamma_{\mu}\right)$. They satisfy the commuting relations

$$
\begin{aligned}
{\left[P_{\mu}, P_{v}\right] } & =0, \quad\left[K_{\mu}, K_{v}\right]=0, \quad\left[M_{\mu v}, D\right]=0, \\
{\left[M_{\mu v}, P_{\lambda}\right] } & =-\left(g_{\mu \lambda} P_{v}-g_{v \lambda} P_{\mu}\right), \quad\left[M_{\mu v}, K_{\lambda}\right]=-\left(g_{\mu \lambda} K_{v}-g_{v \lambda} K_{\mu}\right), \\
{\left[M_{\mu v}, M_{\sigma \rho}\right] } & =g_{\mu \rho} M_{v \sigma}+g_{v \sigma} M_{\mu \rho}-g_{\mu \sigma} M_{v \rho}-g_{v \rho} M_{\mu \sigma}, \\
{\left[P_{\mu}, K_{v}\right] } & =2\left(g_{\mu v} D-M_{\mu v}\right), \quad\left[P_{\mu}, D\right]=P_{\mu}, \quad\left[K_{\mu}, D\right]=-K_{\mu},
\end{aligned}
$$

which are invariant under $P_{\mu} \mapsto-K_{\mu}, K_{\mu} \mapsto-P_{\mu}$ and $D \mapsto-D$.

\section{Twistors as geometric multivectorial elements}

In this section we present and discuss the construction of twistors as algebraic spinors of $C \ell_{4,1}$, using the paravector model, and as elements of $\mathrm{SO}(2 n) / \mathrm{U}(n)$, via the pure spinor formalism.

\subsection{Twistors as algebraic spinors using the paravector model}

The reference twistor $\eta_{\mathbf{x}}$ is defined [12], given $\mathbf{x} \in \mathbb{R}^{1,3}$ and a dotted covariant Weyl spinor ${ }^{5}$ (DCWS) $\Pi=\frac{1}{2}\left(1-i \gamma_{5}\right) \psi=(0, \xi)^{t}$, as the multivector

$$
\eta_{\mathbf{x}}=\left(1+\gamma_{5} \mathbf{x}\right) \Pi
$$

The above expression is an index-free geometric algebra version of Penrose twistor in $\mathbb{R}^{1,3}$, since if a suitable representation ${ }^{6}$ of $\mathbb{C} \otimes C \ell_{1,3}$ is used, we have

$$
\eta_{\mathbf{x}}=\left(1+\gamma_{5} \mathbf{x}\right) \Pi=\left[\left(\begin{array}{cc}
i_{2} & 0 \\
0 & i_{2}
\end{array}\right)+\left(\begin{array}{cc}
-i_{2} & 0 \\
0 & i_{2}
\end{array}\right)\left(\begin{array}{cc}
0 & \vec{x} \\
\vec{x}^{c} & 0
\end{array}\right)\right]\left(\begin{array}{l}
0 \\
\xi
\end{array}\right)=\left(\begin{array}{c}
i \vec{x} \xi \\
\xi
\end{array}\right),
$$

where $\vec{x}=\left(\begin{array}{cc}x^{0}+x^{3} & x^{1}+i x^{2} \\ x^{1}-i x^{2} & x^{0}-x^{3}\end{array}\right)$. The symbol $\vec{x}^{c}$ denotes the $\mathbb{H}$-conjugation of $x$ and $i_{2}:=i \mathbf{1}_{2 \times 2}$.

The adjoint Dirac spinor is defined as $\stackrel{\%}{\psi}=\psi^{\dagger} \gamma_{0}=\left(\bar{\psi}_{1}, \bar{\psi}_{2}, \bar{\psi}_{3}, \bar{\psi}_{4}\right)$ and the transposed twistor

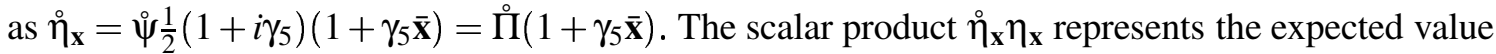
of $\gamma_{5} \mathbf{x}$ with respect to the spinor $\Pi$, since $\stackrel{\circ}{\mathbf{x}}_{\mathbf{x}} \eta_{\mathbf{x}}=\stackrel{\circ}{\Pi} \Pi+2 \stackrel{\circ}{\Pi} \gamma_{5} \mathbf{x} \Pi+\mathbf{x}^{2} \stackrel{\circ}{\Pi} \Pi=2 \stackrel{\circ}{\Pi} \gamma_{5} \mathbf{x} \Pi$. The tensor product $\eta_{\mathbf{x}} \Pi^{\circ}=\left(1+\gamma_{5} \mathbf{x}\right) \Pi \stackrel{\circ}{\Pi}=\left(1+\gamma_{5} \mathbf{x}\right) q$, where $q=\Pi \Pi^{\circ}$ is the chiral positive projection of the timelike vector $Q=\psi \dot{\psi}$, is also presented [12]. It allows to interpret the relation between a twistor, a timelike vector $q$ and the flagpole $\gamma_{5} \mathbf{x} q$, given by the following multivector:

$$
\zeta_{\mathbf{x}}:=\eta_{\mathbf{x}} \stackrel{\circ}{\Pi}=\left(1+\gamma_{5} \mathbf{x}\right) q=q+\gamma_{5} \mathbf{x} q=(1-i \mathbf{x}) q \in C \ell_{4,1} .
$$

\footnotetext{
${ }^{5} \mathrm{~A}$ Weyl spinor can always be written as $\frac{1}{2}\left(1 \pm i \gamma_{5}\right) \psi$, where $\psi$ is a Dirac spinor.

${ }^{6}$ As Keller [12], we choose to use a representation that differs from the Weyl representation by a sign on the matrices representing $\gamma_{1}, \gamma_{2}$ and $\gamma_{3}$.
} 
The incidence relation, that determines a point in spacetime from the intersection of two twistors is defined, leading to the Penrose description $[13,14]$, as

$$
J_{\mathbf{x x}}:=\bar{\eta}_{\mathbf{x}} \eta_{\mathbf{x}}=\stackrel{\circ}{\Pi} \gamma_{5}(\mathbf{x}-\mathbf{x}) \Pi=0 .
$$

The product $J_{\mathbf{x} \mathbf{x}}$ is invariant if $\eta_{\mathbf{x}}$ is multiplied by a complex number. Then eight dimensions are reduced to six, which leads to the classical interpretation of a twistor related to the space $\mathbb{C P}^{3} \simeq$ $\mathrm{SO}(6) /\left(\mathrm{SU}(3) \times \mathrm{U}(1) / \mathbb{Z}_{2}\right)[6,13,14,20]$.

Keller presents another inner product [12], corresponding to the same twistor, but relating distinct points in spacetime, as $J_{\mathbf{x x}^{\prime}}=\bar{\eta}_{\mathbf{x}} \eta_{\mathbf{x}^{\prime}}=\Pi^{\circ} \gamma_{5}\left(\mathbf{x}-\mathbf{x}^{\prime}\right) \Pi$. This product is null if and only if $\mathbf{x}=\mathbf{x}^{\prime}$. The Robinson congruence [13] is defined if we fix $\mathbf{x}$ and let $\mathbf{x}^{\prime}$ vary.

Let $f$ be a primitive idempotent (PI) of $\mathbb{C} \otimes C \ell_{1,3} \simeq C \ell_{4,1}$ and $f_{ \pm}:=\frac{1}{2}\left(1+\mathbf{e}_{3}\right)$ be PIs of $C \ell_{3,0}$. Since the Dirac spinor $\psi$ is an element of the ideal $\left(\mathbb{C} \otimes C \ell_{1,3}\right) f \simeq C \ell_{1,3}^{+} \simeq C \ell_{3,0} \simeq C \ell_{3,0} f_{+} \oplus$ $C \ell_{3,0} f_{-}, \psi$ indeed consists, as well-known, of the direct sum of two Weyl spinors ${ }^{7}$.

Given a paravector $x=x^{0}+x^{A} E_{A} \in \mathbb{R} \oplus \mathbb{R}^{4,1} \hookrightarrow C \ell_{4,1}$ define $\chi=x E_{4} \in \bigoplus_{k=0}^{2} \Lambda^{k}\left(\mathbb{R}^{4,1}\right)$.

Now we define the twistor as an algebraic spinor $\chi \frac{1}{2}\left(1-i \gamma_{5}\right) U f \in\left(\mathbb{C} \otimes C \ell_{1,3}\right) f \simeq C \ell_{3,0}$, where $U$ is a Clifford multivector and so $U f$ is a Dirac spinor. The term ${ }^{8} \Pi:=\frac{1}{2}\left(1-i \gamma_{5}\right) U f=$ $\left(\begin{array}{l}0 \\ \xi\end{array}\right) \in \frac{1}{2}\left(1-i \gamma_{5}\right)\left(\mathbb{C} \otimes C \ell_{1,3}\right)$ is a DCWS. If we take again a basis $\left\{E_{A}\right\}$ of $C \ell_{4,1}$ and a basis $\left\{\gamma_{\mu}\right\}$ of $C \ell_{1,3}$, the isomorphism $C \ell_{4,1} \simeq \mathbb{C} \otimes C \ell_{1,3}$ explicitly given by $E_{0}=i \gamma_{0}, E_{1}=\gamma_{10}, E_{2}=\gamma_{20}, E_{3}=$ $\gamma_{30}$ and $E_{4}=\gamma_{5} \gamma_{0}=-\gamma_{123}$ is useful to prove the correspondence of this alternative formulation with eq.(3.2), and so, with a geometric algebra index-free version of the Penrose classical twistor formalism, by eq.(3.2). Indeed,

$$
\begin{aligned}
\chi \Pi & =\left(x^{0} E_{4}+\alpha^{0} E_{0} E_{4}+x^{1} E_{1} E_{4}+x^{2} E_{2} E_{4}+x^{3} E_{3} E_{4}+\alpha^{4}\right) \Pi \\
& =x^{0}\left(-i \gamma_{0} \Pi\right)+x^{k}\left(\gamma_{k} \gamma_{0}\right)\left(-i \gamma_{0} \Pi\right)+\alpha^{0}\left(i \gamma_{0}\right)\left(-i \gamma_{0} \Pi\right)+\alpha^{4} \Pi \\
& =\left(1+\gamma_{5} \mathbf{x}\right) \Pi=\left(\begin{array}{c}
i \vec{x} \xi \\
\xi
\end{array}\right) .
\end{aligned}
$$

The incidence relation determines a spacetime manifold point if we take $J_{\bar{\chi} \chi}:=\overline{x E_{4} U} x E_{4} U=$ $-\bar{U} E_{4} \bar{x} x E_{4} U=0$, since the paravector $x \in \mathbb{R} \oplus \mathbb{R}^{4,1}$ is in the Klein absolute $(x \bar{x}=0)$.

\subsection{Flagpoles and twistors from pure pinors and spinors}

A generalized flagpole is given by the 2-form $G=\frac{1}{2}\left(\mathfrak{i} \mathfrak{u} \tilde{\mathfrak{u}}-i \mathfrak{u}_{C} \tilde{\mathfrak{U}}_{C}\right)$ [24], where $\mathfrak{u}_{C}$ is the charge conjugation of the pure spinor $\mathfrak{u}$. Given a real vector $p=\left\langle i \mathfrak{u} \mathfrak{u}_{C}\right\rangle_{1}$, corresponding (modulo a real scalar) to a family of coplanar vectors determining the generalized flagpole, let $\omega$ be an element of a maximal totally isotropic subspace of $V$ such that $\omega_{\mathfrak{u}}{ }^{C}=\mathfrak{u}, \omega \mathfrak{u}=0$ and $\left\{\omega, \omega^{*}\right\}=0$. It can be shown that $G=\exp (i \theta) p \omega+\exp (-i \theta) p \omega^{*}$ and $F:=\left.G\right|_{\theta=0}=p\left(\omega+\omega^{*}\right)=\operatorname{Re}(i \mathfrak{u} \tilde{\mathfrak{u}})$ is the Penrose flagpole $[14,24]$.

\footnotetext{
${ }^{7}$ The four types (dotted covariant, undotted covariant, dotted contravariant and undotted contravariant) of algebraic Weyl spinors are indeed elements of the respective minimal lateral ideals $C \ell_{3,0} f_{-}, f_{+} C \ell_{3,0}, f_{-} C \ell_{3,0}$ and $C \ell_{3,0} f_{+}$of the Pauli algebra $C \ell_{3,0}[21,22,23]$.

${ }^{8}$ In order to get a clear correspondence between our formalism and the Keller index-free formulation of twistors, by abuse of notation we adopt the same symbols to describe the DCWS.
} 
Now, from the well-known correspondence between pure pinors and the group $\mathrm{O}(2 n) / \mathrm{U}(n)$ [20], it is possible to adapt the proof of this correspondence, in order to establish the natural correspondence between pure spinors, twistors and the group $\mathrm{SO}(2 n) / \mathrm{U}(n)$.

By definition, a spinor $\mathfrak{u}$ is said to be pure $[3,4]$ if the set $\Xi_{\mathfrak{u}}:=\left\{\alpha \in \mathbb{C}^{2 n}: \alpha(\mathfrak{u})=0\right\}$ has complex dimension $n$. Besides, the natural map from a pure spinor $\mathfrak{u}$ to $\Xi_{\mathfrak{u}}$ induces an equivariant isomorphism from the algebra of pure spinors $\left(\bmod \mathbb{C}^{*}\right)$ to the set $\Xi_{\mathbb{C}}$ of all $n$ dimensional totally null subspaces of $\mathbb{C}^{2 n}$. Now the well-known result proved in [20], asserting that $\Xi_{\mathbb{C}} \simeq \mathrm{O}(2 n) / \mathrm{U}(n)$, permits to link the pure spinors formulation to twistors. Indeed, the product of pure spinors is directly related to $n$-dimensional complex planes [6], which are invariant (mod $\mathrm{U}(1)$ ) under $\mathrm{U}(n)$ actions. Thus it is possible, at least in even dimensions, to identify (via projective pure spinors) a twistor with an element of the group $\mathrm{SO}(2 n) / \mathrm{U}(n)$. In particular, twistors in four and six dimensions are respectively elements of $\mathrm{SO}(4) / \mathrm{U}(2) \simeq \mathbb{C P}^{1}$ and $\mathrm{SO}(6) / \mathrm{U}(3) \simeq \mathbb{C P}^{3}$. The investigation about an analogous mathematical structure and the physical implications of identifying twistors with elements of $\mathrm{SO}(2 n) / \mathrm{U}(n)$ is presented in [6].

\section{Twistors and exceptional structures}

It is well-known that it is possible, at least in three, four, six and ten dimensions, to construct a null vector from spinors. In string twistor formulations some manifolds can be identified with the set of all spinors corresponding to the same null vector, where in a particular case the homogeneous space $\mathrm{SO}(9) / \mathrm{G}_{2}$ arises [25]. Twistors are also an useful tool for the investigation of harmonic maps, as from the Calabi-Penrose twistor fibration $\mathbb{C P}^{3} \rightarrow S^{4}$ [26]. The deep relation between twistors and exceptional structures is illustrated in the classification of compact homogeneous quaternionic-Kähler manifolds, the so-called Wolf spaces [27, 28]. The Wolf spaces associated with exceptional Lie algebras are $\mathrm{E}_{6} / \mathrm{SU}(6) \times \mathrm{Sp}(1), \mathrm{E}_{7} / \mathrm{Spin}(12) \times \mathrm{Sp}(1), \mathrm{E}_{8} / \mathrm{E}_{7} \times \mathrm{Sp}(1)$, $\mathrm{F}_{4} / \mathrm{Sp}(3) \times \mathrm{Sp}(1)$ and $\mathrm{G}_{2} / \mathrm{SO}(4)$. More comments concerning such structures are beyond the scope of the present paper (see [27, 28, 29]).

\section{Concluding remarks}

We presented twistors in Minkowski spacetime as algebraic spinors associated to $\mathbb{C} \otimes C \ell_{1,3}$, using the paravector model, which was also used to describe all the conformal maps as the action of twisted adjoint representations on paravectors of the Clifford algebra over $\mathbb{R}^{4,1}$. The identification of the twistor identified with $\mathrm{SO}(2 n) / \mathrm{U}(n)$ is obtained, from the complex structure based on pure spinors formalism. As particular cases, twistors in four dimensions are elements of $\mathrm{SO}(4)$ modulo the double covering of electroweak group $\mathrm{SU}(2) \times \mathrm{U}(1)$, and in six dimensions twistors are elements of $\mathrm{SO}(6)$ modulo the double covering of the group $\mathrm{SU}(3) \times \mathrm{U}(1)$.

\section{References}

[1] N. Berkovits and L. Motl, Cubic twistorial string field theory, JHEP 04 (2004) 56 [hep-th/0403187].

[2] N. Berkovits and E. Witten, Conformal supergravity in twistor-string theory, JHEP 04 (2004) 9 [hep-th/0406051]. 
[3] E. Cartan, The Theory of Spinors, MIT Press, Cambridge 1967.

[4] C. Chevalley, The Algebraic Theory of Spinors, Columbia Univ. Press, New York 1954.

[5] N. Berkovits, An alternative string theory in twistor space for N=4 super-Yang-Mills, Phys. Rev. Lett. 93 (2004) 11601 [hep-th/ 0402045 ].

[6] N. Berkovits, Pure spinors are higher-dimensional twistors, hep-th/0 0409243.

[7] M. B. Green, J. H. Schwarz and E. Witten, Superstring Theory, vols. I \& II, Cambridge Univ. Press, Cambridge 1987.

[8] W. Baylis, The paravector model of spacetime, in Clifford (Geometric) Algebras with Applications in Physics, Mathematics and Engineering, Birkhäuser, Boston 1995.

[9] I. Porteous, Clifford Algebras and the Classical Groups, Cambridge Univ. Press, Cambridge 1995.

[10] J. Crawford, Clifford algebra: notes on the spinor metric and Lorentz, Poincaré, and conformal groups, J. Math. Phys. 32 (1991) 576.

[11] R. Ablamowicz, Z. Oziewicz and J. Rzewuski, Clifford algebra approach to twistors, J. Math. Phys. 23 (1982) 231.

[12] J. Keller, Spinors, twistors, mexors and the massive spinning electron, Adv. Appl. Cliff. Alg. 3 (1997) 93.

[13] R. Penrose, Twistor algebra, J. Math. Phys. 8, (1967) 345.

[14] R. Penrose and W. Rindler, Spinors and Spacetime, vol.2: Spinor and Twistor Methods in Spacetime Geometry, Cambridge Univ. Press, Cambridge 1986.

[15] A. Crumeyrolle, Orthogonal and Sympletic Clifford Algebras, Kluwer, Dordrecht 1990.

[16] J. Maks, Modulo (1,1) Periodicity of Clifford Algebras and Generalized (anti-)Möbius Transformations, Ph.D. thesis, Technische Universiteit Delft, Delft 1989.

[17] M. F. Atiyah, R. Bott and A. Shapiro, Clifford modules, Topology 3 (1964) 3.

[18] D. Hestenes, The design of linear algebra and geometry, Acta Appl. Math. 23 (1991) 65.

[19] A. Laufer, The Exponential Map, Clifford Algebras, Spin Representations and Spin Gauge Theory of $U(1) \times T^{4}$, Ph.D. Thesis, Universität Konstanz, Konstanz 1997.

[20] F. Reese Harvey, Spinors and Calibrations, Academic Press, Boston 1990.

[21] V. Figueiredo, E. C. de Oliveira and W. A. Rodrigues Jr., Covariant, algebraic, and operator spinors, Int. J. Theor. Phys. 29 (1990) 371.

[22] W. A. Rodrigues, Jr., Algebraic and Dirac-Hestenes spinors and spinor fields, J. Math. Phys. 45 (2004) 2908.

[23] R. A. Mosna and W. A. Rodrigues, Jr., The bundles of algebraic and Dirac-Hestenes spinor fields, J. Math. Phys. 45 (2004) 2945.

[24] I. Benn and R. Tucker, An Introduction to Spinors and Geometry with Applications in Physics, Adam Hilger, Bristol 1987.

[25] M. Cederwall, Introduction to division algebras, sphere algebras and twistors, in proceedings of Theor. Phys. Network Meeting at NORDITA, Kopenhagen, 1993 [hep-th/9310115]. 
[26] P. Z. Kobak, Twistors, nilpotent orbits and harmonic maps, in eds. A.P. Fordy and J.C. Wood, Harmonic maps and integrable systems, Aspects of Mathematics E23, Vieweg, Braunschweig/Wiesbaden 1994.

[27] D. Alekseeviskii, Compact quaternion spaces, Funk. Anal. i Prilozh. 2 (1968) 34; English transl. Funct. Anal. Appl. 2 (1968) 106.

[28] J. Wolf, Complex homogeneous contact manifolds and quaternionic symmetric spaces, J. Math. Mech. 14 (1965) 1033.

[29] P. Claus, M. Gunaydin, R. Kallosh, J. Rahmfeld and Y. Zunger, Supertwistors as quarks of SU(2,214), JHEP 05 (1999) 19 [hep-th/9905112]. 